\title{
Monitoramento Tecnológico e Mercadológico de Biopolímeros
}

\author{
Suzana Borschiver, Luiz F. M. Almeida, Tamar Roitman \\ Escola de Química, UFRJ
}

Resumo: Este artigo aborda um estudo de monitoramento tecnológico em biopolímeros aplicados em diversas áreas, usando como fontes de informações artigos científicos e patentes. Para tanto, foram utilizados, respectivamente, o programa Scifinder Scholar, que emprega as bases de dados CAPLUS e MEDLINE, e a base de dados americana de patentes, a USPTO. Os dados foram obtidos utilizando-se como palavras-chaves "biopolymers" e "biomaterials". Foram realizadas análises "macro", "meso" e "micro" em relação às principais universidades/centros de pesquisa, países de publicação, tipos de aplicações, principais biopolímeros mencionados, principais matérias-primas utilizadas e principais métodos de produção.

Palavras-chave: Biopolímeros, biomateriais, monitoramento tecnológico e mercadológico.

\section{Biopolymers' Technology and Market Monitoring}

Abstract: This paper reports a survey on the technology of biopolymers for various sector, using information extracted from scientific articles and patents. The Scifinder Scholar program based on the CAPLUS and MEDLINE databases and the USPTO databases were used as inputs, respectively. All the data were mined using biopolymers and biomaterials as keywords. The output of this research is a map of biopolymers' university/research centers, origin countries, types of biopolymers and its applications, major raw materials and production methods.

Keywords: Biopolymers, biomaterials, technology and market monitoring.

\section{Introdução}

Os polímeros, cuja produção é estimada na ordem de 180.000.000 t/ano, têm um papel importantíssimo na sociedade moderna, estando presente em praticamente todos os setores da economia como: medicina, agricultura, construção civil, embalagens e eletroeletrônicos ${ }^{[1]}$.

Pode-se considerar o petróleo como uma das fontes principais de matéria-prima para os polímeros, porém com o aumento do seu preço devido aos conflitos geopolíticos no Oriente Médio (principal região produtora de petróleo) e com a crescente preocupação da sociedade com os índices de poluição mundial, fez-se necessário o desenvolvimento de novos tipos de polímeros.

Esses novos tipos de polímeros são chamados biopolímeros e podem ser definidos como qualquer polímero (proteína, ácido nucléico, polissacarídeo) produzido por um organismo vivo. Já os biomateriais são materiais bioativos que têm a capacidade de interagir com tecidos naturais, podendo ser naturais ou sintéticos. A matéria-prima principal para a sua manufatura é uma fonte de carbono renovável, geralmente um carboidrato derivado de plantios comerciais de larga escala como cana-de-açúcar, milho, batata, trigo e beterraba; ou um óleo vegetal extraído de soja, girassol, palma ou de outra planta oleaginosa ${ }^{[1]}$.

Diversos são os polímeros citados na literatura, como por exemplo, o PLA, o PHA, o PA e a Goma Xantana. O PLA (polilactato) que é um poliéster produzido por síntese química a partir de ácido láctico obtido por fermentação bac- teriana de glicose extraído do milho, com uso potencial na confecção de embalagens, itens de descarte rápido e fibras para vestimentas e forrações. O PHA (polihidroxialcanoato) constitui uma ampla família de poliésteres produzidos por bactérias através de biossíntese direta de carboidratos de cana-de-açúcar ou de milho, ou de óleos vegetais extraídos principalmente de soja e palma*. Os PA (polímeros de amido) são polissacarídeos, modificados quimicamente ou não, produzidos a partir de amido extraído de milho, batata, trigo ou mandioca. Pode ser utilizado na produção de embalagens e itens de descarte rápido e, em blendas com polímeros sintéticos, na confecção de filmes flexíveis. A Goma Xantana é um exopolissacarídeo** produzido por microrganismos a partir de carboidratos extraídos de milho ou cana-de-açúcar, com ampla utilização na área de alimentos e uso potencial na de cosméticos e na exploração de petróleo ${ }^{[1]}$.

O mercado dos biopolímeros, exceto a Goma Xantana, era da ordem de 60.000 toneladas em 2002 com preço de comercialização de US\$ 4/kg. Este mercado tem taxa de crescimento acima de $20 \%$ ao ano e custos de produção decrescente para cerca de US\$2/kg, antevendo um consumo em 2015 da ordem 1.000.000 t/ano, perfazendo um mercado anual de US\$ 2 bilhões. É consenso entre as fontes

\footnotetext{
* Além disso, dependendo da composição monomérica, pode ser utilizado na produção de embalagens, de itens de descarte rápido e de filmes flexíveis.

** Os exopolissacarídeos, chamados de biopolímeros ou gomas hidrossolúveis, são produzidos por uma grande variedade de microrganismos e possuem propriedades físicas, estruturais e químicas bastante homogêneas, em relação aos derivados de algas e plantas.
} 
consultadas que o preço de venda do produto é o obstáculo principal para sua entrada no mercado, apontando-se um preço teto de cerca de US\$2/kg, ou seja, cerca de até $50 \%$ superior aos preços de comercialização das resinas sintéticas mais comuns (PE, PP, PET). Não obstante, entre os anos 1990 e 2002 houve duplicação da demanda de bioplásticos a cada dois anos.

Uma análise da evolução do preço destes biopolímeros, levando-se em conta a curva de evolução tecnológica e do preço do petróleo em diversos cenários, demonstrou que os preços de polietileno tereftalato (PET) e bioplásticos convergiriam para cerca de US\$1/kg no ano de 2020, caso o petróleo subisse de US\$ 25 a US\$ 50/barril, cenário bastante provável na atual conjuntura. A Goma Xantana tem um mercado atual de cerca de US\$ 270 milhões e 40.000 t/ano com taxa de crescimento acima de 5\% ao ano, perfazendo em 2015 um mercado de US\$ 400 milhões e 80.000 t/ano ${ }^{[1]}$.

Devido a essa grande importância fez-se necessário uma pesquisa nessa área, resultando nesse trabalho que tem como objetivo realizar um monitoramento tecnológico e mercadológico da área de biopolímeros em geral.

\section{Metodologia}

Este estudo faz parte de um projeto de pesquisa em andamento, sobre prospecção tecnológica de biopolímeros, que está estruturado em diversas etapas como, monitoramento tecnológico em artigos, em patentes e utilização de software de gestão. Nesse estudo é apresentado um monitoramento em artigos e patentes.

A prospecção e o monitoramento informacional são etapas fundamentais do processo de inteligência competitiva (IC). Através da prospecção informacional é possível estabelecer um mapa inicial de fontes de informação e conhecimento essenciais a uma maior competitividade de um determinado setor. O mapeamento de dados, informação e conhecimento, alimentam os diferentes sistemas informacionais existentes, estabelecendo uma dinâmica na entrada dos dados, informação e conhecimento.

O monitoramento precisa selecionar cuidadosamente, dentre um grande número de informações, aquelas que têm potencial relevância, assim como deve funcionar como uma antena na identificação de novas oportunidades e sinais de mudança no ambiente.

\section{Monitoramento tecnológico em artigos}

O monitoramento tecnológico foi realizado através da base Scifinder Scholar que é um programa que inclui referências e resumos de periódicos, patentes, livros, relatórios técnicos, teses e dissertações e como complementação, através de pesquisa in loco na biblioteca do Instituto de Macromoléculas Professora Eloísa Mano, da Universidade Federal do Rio de Janeiro (IMA/UFRJ).

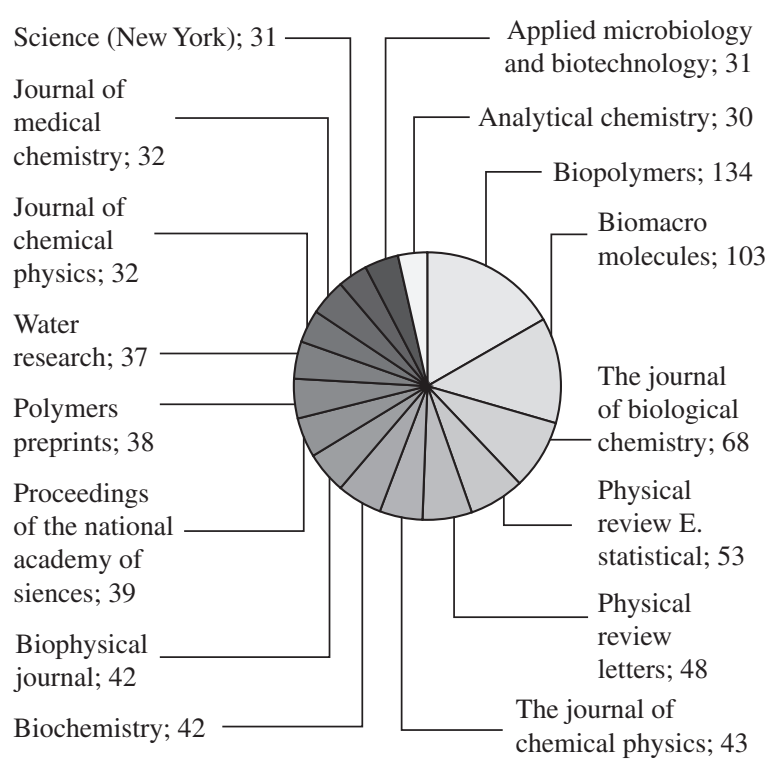

Figura 1. Principais revistas que publicaram artigos sobre Biopolimeros entre 2002-julho/20007.

No IMA, foram consultados bibliografias especializadas no intuito de esclarecer os termos técnicos encontrados nos $\operatorname{artigos}^{[2-4]}$.

No programa SciFinder que tem como recurso as bases de dados CAPLUS e MEDLINE, as palavras-chaves empregadas na busca foram "biopolymers" e "biomaterials". $\mathrm{Na}$ busca foram considerados apenas artigos publicados em revistas cientificas entre 2002 e 2007, encontrando-se um total de 3838 artigos, já com a remoção das duplicatas.

No sentido de racionalizar a pesquisa, foi realizada uma nova busca utilizando-se a mesma base de dados, porém foram selecionadas somente as revista que tinham mais de 30 publicações, conforme Figura 1. Assim, o número de artigos reduziu-se para 803 , sendo que apenas 220 falavam especificamente sobre biopolímeros.

Esses artigos foram analisados mais detalhadamente em relação ao ano de publicação, instituição de pesquisa, países a que pertencem essas instituições, biopolímeros mencionados, assim como suas aplicações, tipo de matéria-prima e produção.

\section{Resultados e Discussão}

Foram realizadas análises "macro", "meso" e "micro" em relação aos resultados obtidos.

\section{Análise macro}

\section{Distribuição de artigos por ano}

$\mathrm{Na}$ análise por ano de publicação percebe-se através da Figura 2 que em 2006 houve o maior número de artigos publicados. 


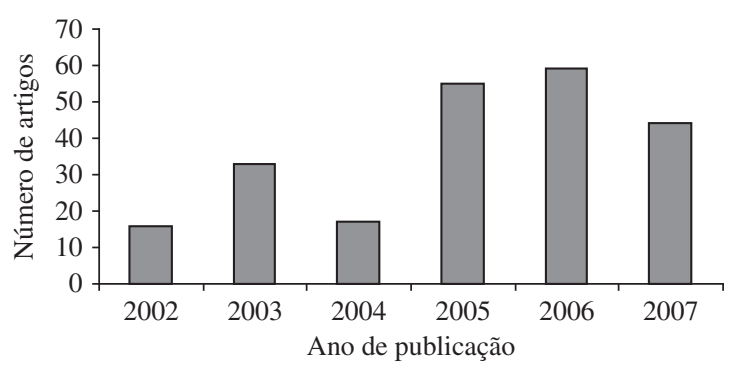

Figura 2. Evolução do número de artigos publicados sobre biopolimeros entre 2002-2007.

\section{Distribuição de artigos por instituição}

As instituições que publicaram artigos relacionados a biopolímeros foram classificadas em empresas, universidades/centros de pesquisa e pessoa física. Dentre essas, as/ os universidades/centros de pesquisa foram as que mais se destacaram no número de publicações. Essa distribuição é representada na Figura 3.

A Tabela 1 a seguir mostra as/os principais universidades/ centros de pesquisa, que estão presentes na tabela centros de pesquisa/universidades com, no mínimo, quatro artigos pu-

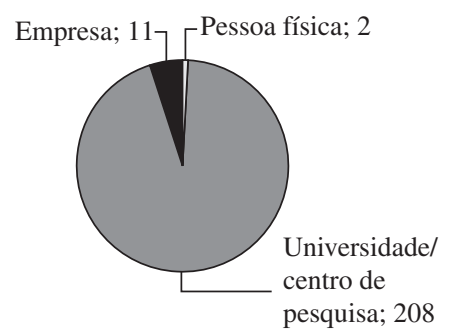

Figura 3. Distribuição por tipo de instituição entre 2002-julho/2007.

Tabela 1. Principais centros de pesquisa.

\begin{tabular}{cc}
\hline Principais centros de pesquisa & $\begin{array}{c}\text { Número de } \\
\text { publicações }\end{array}$ \\
\hline Russian Academy of Sciences & 5 \\
University of California & 5 \\
University of Massachusetts & 5 \\
Worcester Polytechnic Institute & 5 \\
University of Cambridge & 5 \\
University of Washington & 5 \\
National Institute of Health & 4 \\
Chinese Academy of Sciences & 4 \\
University of Toronto & 4 \\
Westfaelische Wilhelms-Universitaet & 4 \\
Muenster & \\
Michigan State University & 4 \\
Academy of Sciences of the Czech & 4 \\
Republic & 4 \\
Seoul National University & 4 \\
University of Leeds & 4 \\
University of Pittsburgh &
\end{tabular}

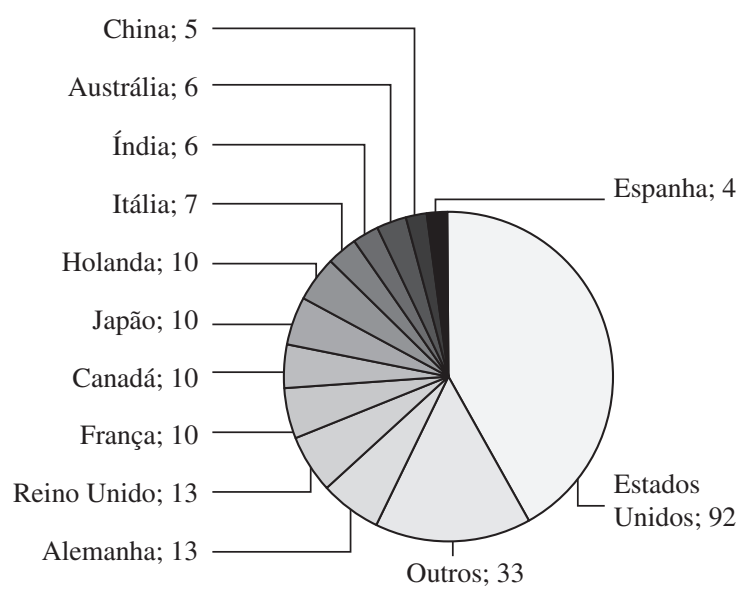

Figura 4. Distrubuição dos artigos publicados por países entre 2002julho/2007.

blicados. O número total de centros de pesquisa/universidades foi de 59.

Em relação ao país de origem das instituições, os Estados Unidos se destacaram, com $42 \%$ do total de publicações. Pela Figura 4, percebe-se uma concentração maior desse tipo de pesquisa nos países desenvolvidos.

\section{Análise meso}

\section{Biopolímeros mencionados}

Foram identificados 13 diferentes tipos de biopolímeros e a Figura 5 mostra a distribuição por números de artigos. Sobre os tipos de biopolímeros pode-se destacar o PHA, o Dextran, a Goma Xantana e o PHB (polihidroxibutirato). O PHB pode ser usado na fabricação de embalagens para produtos de limpeza e de higiene, cosméticos e produtos farmacêuticos. Além disso, por ser biocompatível e facilmente absorvido pelo organismo humano, pode ser empregado na área médico-farmacêutica, prestando-se à fabricação de fios de sutura, próteses ósseas e cápsulas que liberam gradualmente medicamentos na corrente sanguínea ${ }^{[5]}$. Também foram mencionadas algumas blendas como o PU, que são misturas de biopolímeros com polímeros sintéticos.

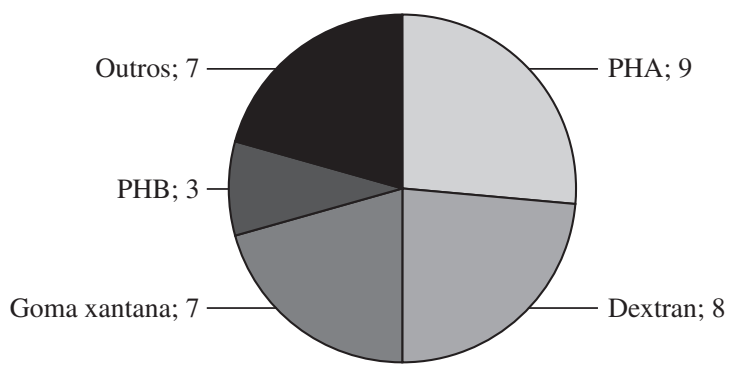

Figura 5. Distribuição dos biopolímeros pelo número de artigos entre 2002julho de 2007. 


\section{Análise micro}

\section{Distribuição por aplicações}

A distribuição por aplicações foi realizada em oito grupos e está representada na Figura 6. A principal aplicação mencionada de biopolímeros ocorre na Área Medicinal, e dentro dessa, as aplicações com maior destaque foram implantes com 2 artigos, terapia genética com 2 artigos, engenharia de tecidos com 2 artigos e cápsulas para comprimidos com 3 artigos. As outras áreas de destaque encontradas foram as de Nanotecnologia, com 10 artigos, em que dois deles discorreram sobre microtubos, e a área da Indústria de Biotecnologia com 8 artigos sendo 2 sobre biosensores.

\section{Distribuição por tipo de matérias-primas}

A Figura 7 a seguir ilustra a divisão por tipos de matériasprimas empregadas na produção dos biopolímeros. A principal matéria-prima encontrada foi a celulose. Isso pode ser explicado pela grande disponibilidade desse polímero.

\section{Distribuição por métodos de produção}

Dentre as formas de se produzirem biopolímeros, apresentadas na Figura 8 destacaram-se a síntese por bactérias e encadeamento de peptídeos. As bactérias mencionadas foram: Shewanella oneidensis, Pseudomonas oleovorans, Ralstonia eutrofa, Methylobacteriu e Halomonas eurihalina.

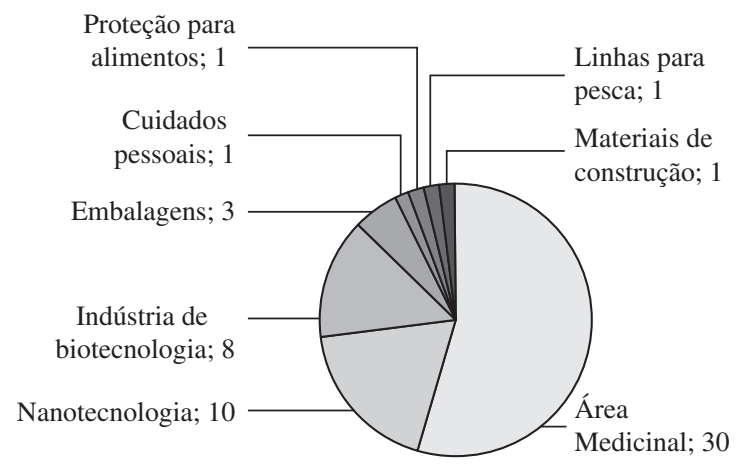

Figura 6. Distribuição das patentes por área de aplicação do biopolímero.

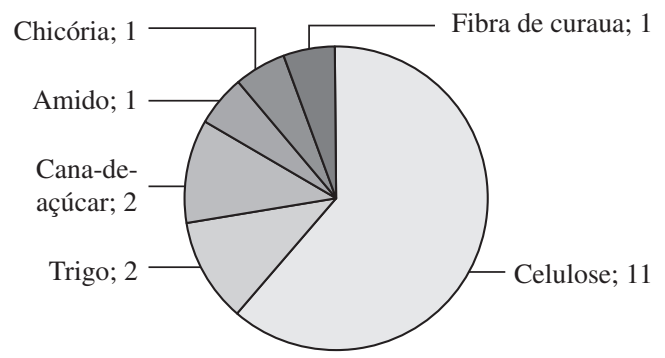

Figura 7. Distribuição dos tipos de matérias-primas pelo número de artigos onde são mencionadas entre 2002-julho/2007.

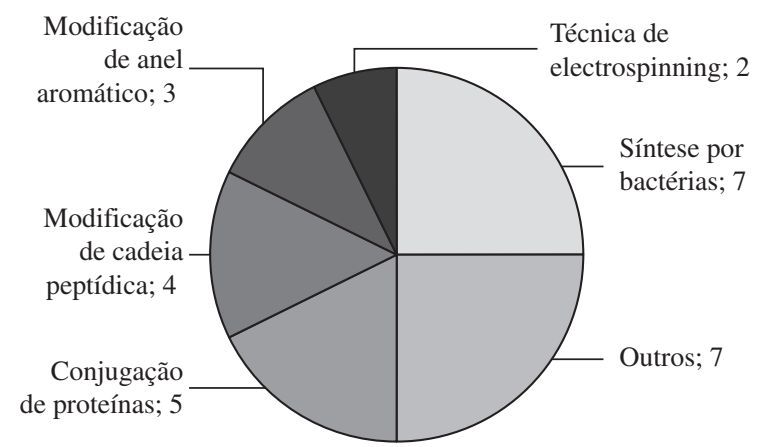

Figura 8. Distribuição dos tipos de processos para a produção de biopolímeros entre 2002-julho/2007.

\section{Monitoramento Tecnológico em Patentes}

A base de dados utilizada como fonte de informação foi a USPTO. Os termos empregados como palavras-chave foram "Biopolymers" e "Biomaterials" e a busca restringiu-se às patentes que as continham no título e no resumo. Foram encontrados 119 patentes entre os anos de 2001 a 2007, mas removendo-se as duplicatas, o número passou para 107.

Essas patentes foram analisadas mais detalhadamente da mesma forma realizada com os artigos.

Como já mencionado, o espaço de tempo abrangido na análise foi do ano de 2001 até o mês de outubro de 2007. Através da Figura 9, é possível perceber um crescente depósito de patentes relacionadas ao assunto e observa-se que o ano de 2006 apresentou o maior número de patentes depositadas.

\section{Distribuição por país}

A distribuição por países depositantes das patentes registradas apontou os Estados Unidos como o principal responsável pelos registros de patentes envolvendo nanotecnologia, respondendo por $43 \%$ do total das patentes. Em segundo lugar está a Alemanha, com 19\% e o Japão em terceiro com $12 \%$. Os demais representam $5 \%$ ou menos do total, como mostra a Figura 10.

\section{Analise por instituição}

Como já mencionado anteriormente, uma das análises foi realizada em relação aos principais depositantes, classificados em empresas, universidades/centros de pesquisa e pessoa física. No caso das patentes, o depósito feito por pessoas fí-

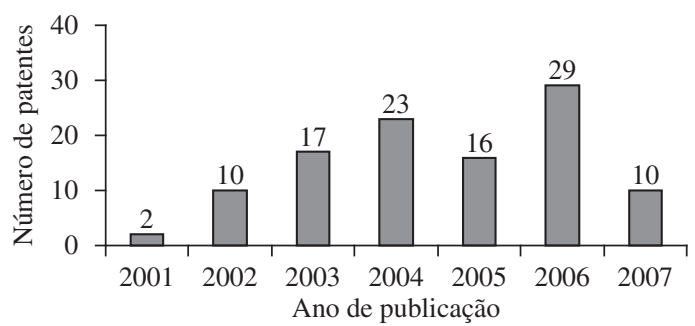

Figura 9. Distribuição das patentes por ano de publicação. 
sicas obteve maior destaque, com $64 \%$ do total, como visto na Figura 11.

\section{Análise meso}

\section{Biopolímeros mencionados}

Nos textos, foram citados 51 tipos de biopolímeros e biomateriais. Dentre eles, os que mais apareceram foram o colágeno e a Chitosana. A Tabela 2 a seguir relaciona a quantidade de citações em patentes com os biopolímeros/biomateriais, apresentando apenas os que aparecem em, pelo menos, dois textos.

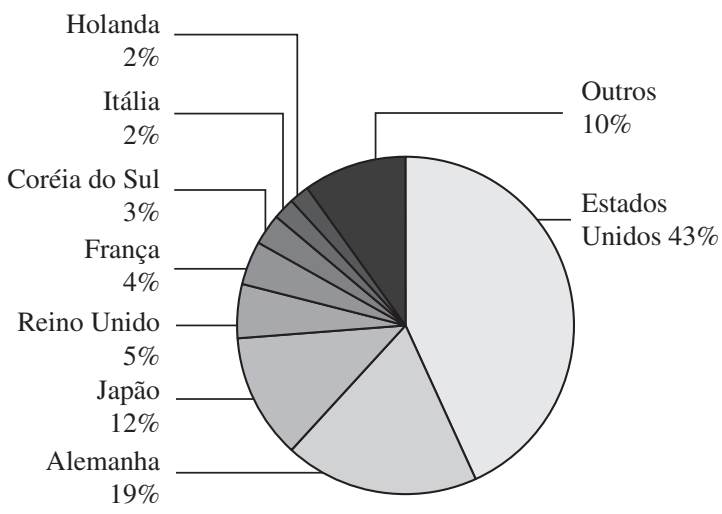

Figura 10. Distribuição das patentes por países depositantes.

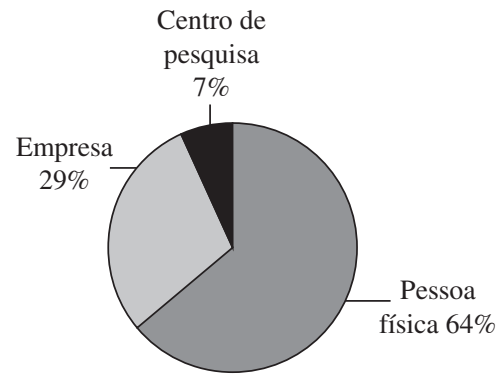

Figura 11. Distribuição das patentes por tipo de instituição.

Tabela 2. Principais biopolímeros mencionados.

\begin{tabular}{cc}
\hline Biopolímeros mencionados & $\begin{array}{c}\text { Número de } \\
\text { patentes }\end{array}$ \\
\hline Colágeno & 9 \\
Chitosana & 5 \\
Chitin & 3 \\
Ácido hialurônico & 3 \\
Amido & 2 \\
Poliuretano & 2 \\
Goma xantana & 2 \\
Elastin & 2 \\
Keratin & 2 \\
\hline
\end{tabular}

Análise micro

\section{Distribuição por aplicações}

- Medicinal: 31 patentes;

- Composições farmacêuticas: 1 patente;

- Imobilização de enzimas: 1 patente;

- Materiais superabsorventes: 1 patente;

- Tecnologia para alimentos: 1 patente;

- Tintas para cabelo: 1 patente;

- Sistemas de controle químico: 1 patente; e

- Fabricação de biosensores: 1 patente.

\section{Distribuição por métodos de produção}

- Cross-link: 5 patentes;

- Matrizes para a extração, purificação, separação e armazenamento de biopolímeros: 3 patentes;

- Síntese através da diminuição de forças dos grupos protetores: 3 patente;

- Irradiação do material: 2 patentes;

- Tratamento com agentes de revestimento do biomaterial: 2 patentes;

- Obtenção a partir de tecidos ósseos/tendão: 2 patentes;

- Produção de hidrogel por iniciação química ou fotopolimerização: 1 patente;

- Produção de Biopolímeros através da síntese de substrato: 1 patente;

- Produção de Biopolímeros via catalise enzimática: 1 patente;

- Produção por sujeitar leveduras a um tratamento de pressão: 1 patente;

- Filtração de biopolímeros pela pressão de campo elétrico: 1 patente;

- Produção de biopolímeros pela irradiação sólida na presença gases insaturados: 1 patente;

- Produção de biopolímeros atraves de arranjos de luz de diodo: 1 patente;

- Extração de biopolímeros de uma biomassa usando líquidos iônicos: 1 patente;

- Exposição do biomaterial a uma solução contendo álcool por 24 horas pelo menos: 1 patente;

- Imersão de matriz de vidro em solução contendo nutriente inoculado: 1 patente;

- Tratamento químico de tecido de mamíferos: 1 patente; e

- Anodização de silício: 1 patente.

\section{Conclusão}

A partir do estudo dos artigos científicos publicados entre os anos de 2002 e 2007 e das patentes depositadas entre 2001 e 2007, pode-se obter algumas conclusões:

- Analisando as publicações e depósitos por ano, podese verificar que houve um aumento gradual do número de textos sobre o tema, e o ano de 2006 apresentou o 
maior número de publicações de artigos e de depósitos de patentes;

- Em relação aos países, observa-se que Estados Unidos e Alemanha aparecem em destaque tanto na publicação de artigos quanto no depósito de patentes. As universidades/centros de pesquisa tiveram o maior número de publicações de artigos, enquanto as pessoas físicas depositaram mais patentes. Entre as empresas, vale destacar a dinamarquesa a Danisco Company e a americana Biomass Processing Technology, Inc.;

- Dentre os biopolímeros, os que mais se destacaram nos artigos foram o PHA, o Dextran e a Goma Xantana, somando $84 \%$ do total, e nas patentes foram o colágeno e a Chitosana, com $24 \%$ do total;

- As aplicações de biopolímeros são muito amplas e estão em constante desenvolvimento, principalmente nas áreas medicinal e de nanotecnologia. Vale a pena destacar sua grande importância dentro da medicina como na utilização em engenharia de tecidos e implantes, assim como na regeneração de ossos, tema muito explorado nos artigos publicados no Congresso Internacional The Polymer Processing Society - 23 Anual Meeting realizado em Salvador, Bahia em 2007;

- A principal matéria-prima utilizada para a produção de biopolímeros foi a celulose, com destaque também para trigo e amido; e
- Em relação ao processo de produção de biopolímeros, pode-se destacar a síntese por bactérias, o encadeamento de peptídeos e o cross-link.

\section{Referências Bibliográficas}

1. Pradella, J. G. C. - "Biopolímeros e Intermediários Químicos", relatório técnico nº 84396-205, Centro de Tecnologia de Processos e Produtos, Laboratório de Biotecnologia Industrial - LBI/CTPP (2006).

2. Shalaby, S. W.; Ikada, Y.; Langer, R. \& Williams, J. "Polymers of Biological and Biomedical Significance”, American Chemical Society, Washington (1994).

3. Dumitriu, S. - "Polimeric Biomaterials", Marcel Dekker, Inc., New York (1994).

4. Salamone, J. C. - "Concise-Polymeric Materials Encyclopedia”, CRC Press, Boca Raton (1999).

5. Coutinho, et al. - "A importância e as vantagens do Polihidroxibutirato (plástico biodegradável)", Holos Environmet, Ano 20, dezembro de 2004.

Enviado: 24/09/07

Reenviado: 05/04/08

Aceito: 09/05/08 